\title{
An Algorithm Based on Relax for Radar HRRP Feature Extraction
}

\author{
Shen Limin* \\ The PLA University of Information Engineering Luoyang Campus, Luoyang Henan 471009, China
}

\begin{abstract}
Due to the complexity of target scattering points in high-resolution range cell, the paper makes a detailed study of the scattering center model based on the theory of the scattering center model, and analyzes the traditional extraction algorithm's limitations. An improved feature extraction method based on Relax for feature extraction has been presented in this paper. Firstly, the method has preprocessed the original digitals so that the noises can be cleaned up and the useful information can be strengthened, and then extracted features in effective range cell. Simulation results based on the measured data prove that the new method could reduce the computation complexity and increase the recognition rate, accurately reflecting geometry positional information of the target.
\end{abstract}

\section{Introduction}

Radar target recognition based on High Resolution Range Profile (HRRP) is the mainstream of the modern war environment perception and recognition. Radar can work effectively throughout the day, all-weather, and long-distance, and is therefore used as the main detection method for battlefield perception. Radar target recognition based on HRRP avoids the complicated motion compensation and can greatly improve the target interception ability to meet the requirements of real-time guidance. HRRP recognition is an important method for the identification of complex military targets and has received extensive attention and in-depth research. Antinoise performance, azimuth sensitivity, and training-set size sensitivity are important indicators of the overall performance of the HRRP classifier ${ }^{[1,2]}$.

Feature extraction is the most critical for radar target recognition. The recognition ability of target recognition system depends greatly on the selection of feature vectors and the accuracy of feature extraction. At present, the feature extraction method based on HRRP is mainly divided into non-parametric methods and parameterized methods ${ }^{[3,4]}$. The typical representation of nonparametric methods is the direct use of HRRP. Li and Yang elaborated the feasibility of directly using HRRP as feature vectors, and proposed a recognition method based on matching degrees. The typical representation of the parametric method is the Relax algorithm based on the simple scattering point model, this algorithm is based on the Clean algorithm[ ${ }^{5-7]}$. Based on the Relax algorithm, this paper presents an improved feature extraction method, which uses the position and intensity of the scattering center as the target feature to extract the geometric parameters of the target structure.

\section{Target scattering point model}

The simple scattering point model theory shows when the radar work in optical region, the target can be composed of several discrete scattering points, and the radar echo is the vector sum of the sub-echo formed by the backscattering of the emission point by each scattering point ${ }^{[7-9]}$. Relax algorithm is a nonlinear least squares relaxation algorithm for solving simple scattering point model parameters. This paper extracts features, using the average distance of the Relax algorithm for radar echo. The simple scattering point model of radar target with noise is.

$$
y_{n}=\sum_{k=1}^{K} \alpha_{k} e^{j 2 \pi f_{k} n}+e_{n}
$$

$k$ represents the number of scattered points of radar exposure at a specific target azimuth; $a_{k}$ is the complex envelope of the kth scattering point echo, which is a function of time and represents the radar target intensity; $f_{k}$ is the kth sine wave frequency, which means the distance to the position information. Assuming $e_{n}$ is white noise, in the Relax algorithm, the noise relaxation is the self-regression process, ie:

$$
e_{n}=\sum_{m=1}^{M} a_{m} e_{n-m}+w_{n} \quad n=0,1, \cdots N-1
$$

$w_{n}$ represents a zero-mean complex Gaussian white noise process. Making:

$$
\begin{gathered}
y=\left[y_{0}, y_{1}, \cdots y_{N-1}\right]^{T} \\
w\left(f_{k}\right)=\left[1, e^{j 2 \pi f_{k}}, e^{j 2\left(2 \pi f_{k}\right)}, \cdots, e^{j(N-1) 2 \pi f_{k}}\right]^{T} \\
w=\left[w\left(f_{1}\right), w\left(f_{2}\right), \cdots, w\left(f_{K}\right)\right]
\end{gathered}
$$

\footnotetext{
* Corresponding author: shenlimin1996@163.com
} 


$$
a=\left[a_{1}, a_{2}, \cdots a_{K}\right]^{T}
$$

Rewrite the above model in matrix form.

$$
Y=A \alpha+e_{n}
$$

The problem is to solve the parameters based on the above model and radar data, ie

$$
\left\{f_{k}, a_{k}\right\}=\arg \min \underset{\left\{f_{k}, a_{k}\right\}}{\arg \min }\|Y-A \alpha\|
$$

\section{An improved Relax algorithm}

The idea of algorithm improvement mainly focuses on the selection of effective distance units, it can be considered from two aspects: firstly there are almost no scattering point echoes on some distance units, or there are only some very weak clutter and noise; Secondly, there are many scattering points in some distance unit distribution, but there is no obvious special point. Let variable integer $P$ represent the value of the distance unit and the initial value is 1 . If any one of the above two conditions is satisfied, $\mathrm{P}$ is set to 0 , not estimating the parameter of scattering points. The algorithm can be done only for the effective distance to improve the efficiency of feature extraction.Assuming the parameters $\left\{a_{k}, f_{k}\right\}$ have been estimated, Signal $\sum_{i=1, i \neq k}^{K} a_{i} w\left(f_{i}\right)$ reconstructed from a simple scatter point model has a residual signal of:

$$
\begin{gathered}
y_{k}=Y-\sum_{i=1, i \neq k}^{K} a_{i} w\left(f_{i}\right)=Y-w a+w\left(f_{k}\right) a_{k} \\
a_{k}=\frac{w^{T}\left(f_{k}\right) y_{k}}{N}=\frac{D F T\left(y_{k}\right)}{N} \\
f_{k}=\arg \max _{f_{k}} \arg \left\|\operatorname{DFT}\left(y_{k}\right)\right\|^{2}
\end{gathered}
$$

The algorithm implementation steps are as follows:

Initialization: read HRRP data and set the number of scattered points

Step1: let $k=1$,Calculating $a_{l}$ and $f_{l}$ using formula (6) and (7), then $p+1$

Step2: (1)let $k=2$, Calculating $y_{1}, y_{2}$ using formula (5) and $\left\{a_{1}, f_{1}\right\}$, Calculating $\left\{a_{2}, f_{2}\right\}$ using formula (6) and (7), then $p+1$;

(2)Get $y_{1}$ from $\left\{a_{2}, f_{2}\right\}$ and formula (5), recalculating $\left\{a_{1}, f_{1}\right\}$ from formula (6),(7) and $y_{1}$;

(3)Get $y_{2}$ from $\left\{a_{1}, f_{1}\right\}$ and formula (5) and $\left\{a_{1}, f_{1}\right\}$, recalculating $\left\{a_{2}, f_{2}\right\} \quad$ from formula (6),(7) and $y_{2}$,Repeat (2) and (3) until the control convergence condition is satisfied.

Step3: (1)let $k=3$,Calculating $y_{3}$ using formula (5) and $\left\{a_{i}, f_{i}\right\}_{i=1, i=2}$, Calculating $\left\{a_{3}, f_{3}\right\}$ using formula (6) and (7), then $p+1$;
(2)Get $y_{1}$ from $\left\{a_{i}, f_{i}\right\}_{i=2, i=3}$ and formula (5) recalculating $\left\{a_{1}, f_{1}\right\}$ from formula (6),(7) and $y_{1}$;

(3)Get $y_{2}$ from $\left\{a_{i}, f_{i}\right\}_{i=1, i=3}$ and formula recalculating $\left\{a_{2}, f_{2}\right\}$ from formula (6),(7) and $y_{2}$;

(4)Get $y_{3}$ from $\left\{a_{i}, f_{i}\right\}_{i=1, i=2}$ and formula (5), recalculating $\left\{a_{3}, f_{3}\right\}$ from formula (6),(7) and $y_{3}$,Repeat (2) to (4) until the control convergence condition is satisfied. According to the above method, calculate the parameter $\left\{a_{k}, f_{k}\right\}$ value when $k=4,5, . . k$.

\section{Experimental result analysis}

In order to verify the effectiveness of the proposed algorithm, the HRRP of the radar target C-130 with pitch angle and horizontal azimuth spacing is simulated. The influence of the number of scattered points and the data processing speed are verified.

\subsection{Influence of scattering points}

The experimental results are shown in Fig1, as follows (a), (b), (c), and (d) are reconstructed from the scattered points of $30,20,15$ and 8 respectively.

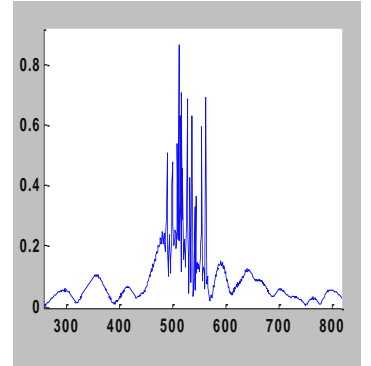

(a)

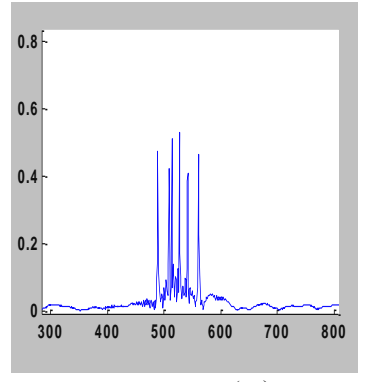

(c)

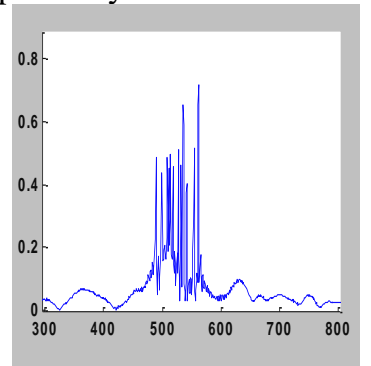

(b)

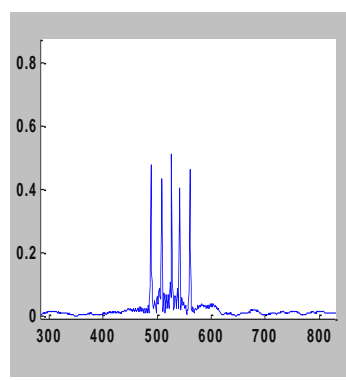

(d)
Fig. 1. The restructuring HRRP of choosing different scattering spots.

It can be seen from the results that the target scattering point parameters extracted by the RELAX algorithm can reflect the scattering point distribution of the original range profile. From the simulation diagram, the more the scattering points are extracted from the target echo, the closer the reconstruction range profile is to the original range profile, and more beneficial to the design of the classifier. It can be seen from fig. 1 (c) and (d) that when the number of scattered points is less than 
15 , the complete distance image cannot be constructed. Fig. 1(a), (b) When the number of scattering points are greater than 20 , the reconstructed range profile has a small difference from the original range profile.

\subsection{Processing speed}

Table 1 shows the simulation results from two aspects of processing time and correct recognition rate. It can be seen from Table 1 that the improved algorithm has considerable advantages, Therefore, the improved algorithm fulfils the dual requirements of the real-time and recognition rate for radar target recognition.

Table 1. Compared the performance with Relax and improved Relax

\begin{tabular}{|c|c|c|c|c|c|}
\hline & scattering points & 30 & 25 & 15 & 10 \\
\hline $\begin{array}{c}\text { Relax } \\
\text { algorithm }\end{array}$ & Processing time(s) & 15.01 & 13.01 & 5.03 & 3.18 \\
\cline { 2 - 6 } & $\begin{array}{c}\text { Correct } \\
\text { recognition rate }\end{array}$ & 0.97 & 0.96 & 0.96 & 0.93 \\
\hline \multirow{2}{*}{$\begin{array}{c}\text { improved } \\
\text { Relax }\end{array}$} & Processing time(s) & 12.36 & 10.58 & 3.26 & 2.07 \\
\cline { 2 - 6 } & $\begin{array}{c}\text { Correct } \\
\text { recognition rate }\end{array}$ & 0.98 & 0.97 & 0.97 & 0.95 \\
\hline
\end{tabular}

\section{Conclusion}

This paper first analyzes the data model of the target echo signal. Based on this model, Relax algorithm is deeply analyzed. The Relax algorithm is improved for the defects of the Relax algorithm of the scattering point. with the improved Relax algorithm, the features of scattering point parameters have been extracted for the measured data on target $\mathrm{C}-130$ radar echo and established the simulation system based on MATLAB to complete the target $\mathrm{C}-130$ radar echo. The different scattering points have been extracted and the range profile has been reconstructed. Finally, the simulation results show that the improved algorithm is effective in selecting the number of scattered points and the speed of data processing.

\section{References}

1. Deshuang.Huang, Technology of Intelligent Signal Processing Based On High Resolution Radar[M].Beijing: Publishing House Of Machinery Industry,(2008)

2. H-J.Li,Y.D,Wang,L-H.Wang,Matchingscore properties between range profile of high resolution radar targets, IEEE Antennas and Propagation Magazine[J]. 44(4): 444-452,(1996)

3. Xing $\mathrm{M}, \mathrm{Wu} \mathrm{R}$, Lan $\mathrm{J}$, et al.Migration Through Resolution Cell Compensation in ISAR Imaging[J].IEEE GRS.Letters , 1(2) : 360400,(2004)

4. Bao zheng,Xing Mengdao,Wang tong. Radar Imaging Technique[M] Beijing: Publishing House Of Electronics Industry. (2015)
5. Bingnan.Pei, Study of High Range Resolution Radar Automatic Target Recognition [D],XI AN: Xidian University.(2002)

6. Hongda Yao. Study of Radar Target Recognition using Range Profiles Based on Time Frequency Features and Kernel Methods [D],ChengDu: University of Electronic Science and Technology of China.(2007)

7. Du Lan. Study on Radar HRRP Target Recognition[D]. XI AN : Xidian University.(2007)

8. Limin Shen .Study of Radar Target Recognition Method Based on High Range Resolution Profile [D].LuoYang: Henan University of Science and Technology.(2014)

9. Chen feng.Improvement on Radar Autiomatic Target Recognition Using HRRPs[J].Beijing:Journal of Electronics \& Information Technology Vol.29 No.6,(2017) 